\title{
Embryonic Development of Axon Pathways in the Drosophila CNS. I. A Glial Scaffold Appears Before the First Growth Cones
}

\author{
J. Roger Jacobs and Corey S. Goodman \\ Department of Biochemistry, Howard Hughes Medical Institute, University of California, Berkeley, Berkeley, California \\ 94720
}

Three classes of glial cells are present early in embryogenesis and appear to play a major role in axon pathway formation in the Drosophila CNS. Six longitudinal glial (LG) cells are present over the longitudinal connective on each side of each segment. Six midline glia (MG) cells surround the anterior and posterior commissures of each segment. Finally, the intersegmental nerve root is covered by a glial cell: the segment boundary cell (SBC). All 3 classes of glial cells are present in their final position before axon outgrowth and their pattern prefigures the first axon pathways. The pioneer growth cones that establish the first axon pathways in the longitudinal connective and intersegmental nerve extend along the elongate surface of the LG and SBC glial cells; the pioneer growth cones for the anterior and posterior commissures extend toward and make close contact with the end feet of the MG glial cells. Later, all 3 classes of glial cells enwrap the axon tracts in much the same way as vertebrate oligodendrocytes.

The results suggest that these early glial cells provide guidance cues for the first growth cones in the Drosophila CNS. More than simply providing a permissive substrate, the differential extension of specific early growth cones towards either the MG cells or along the LG cells suggests an active role for these glia in growth cone guidance.

Glia are prominent cellular components of both vertebrate and invertebrate nervous systems. They occur in a wide variety of morphological types and appear to subserve a wide variety of supporting roles in the mature nervous system, including being conveyors of nutrients and metabolites, providers of electronic insulation, and maintainers of ionic homeostasis. Vertebrate glia have been subdivided into 4 basic classes (astroglia, oligodendroglia, microglia, and Schwann cells) based upon morphological, biochemical, and functional criteria (see Roitbak, 1983, for review). Although invertebrate glia have not been as easy to classify based on morphological criteria alone, many workers nevertheless have described several different types of invertebrate glia, presumably subserving a similar variety of supportive roles (Wigglesworth, 1959; Strausfeld, 1976; Hoyle, 1986).

In addition to their roles in the mature nervous system, glia

\footnotetext{
Received Sept. 20, 1988; revised Nov. 29, 1988; accepted Dec. 5, 1988.

We thank Nipam Patel for Figure $1 A$, P. Borin for Figure 7, Frances Thomas for technical assistance, and John Stevens for access to serial reconstruction equipment. This work was supported by a Canadian MRC Fellowship (J.R.J.) and the Howard Hughes Medical Institute and NIH Grant HD21294 (C.S.G.).

Correspondence should be addressed to J. Roger Jacobs at the above address. Copyright (c) 1989 Society for Neuroscience $0270-6474 / 89 / 072402-10 \$ 02.00 / 0$
}

have also been shown to have important roles during the development of the nervous system, particularly as a permissive, and sometimes active, substrate for migrating neurons and extending growth cones. Much of what is known about the differentiation and role of glia during CNS development has emerged from studies on vertebrate nervous systems, for example, from studies on the cerebellum and corpus callosum in the mouse (Rakic, 1971; Silver et al., 1982). In both systems, glial substrates are present before neuronal differentiation and act as a necessary substrate for neuronal migration or axon outgrowth. In a few isolated cases, a similar role for invertebrate glia in axonogenesis (Bastiani and Goodman, 1986) and in regeneration (Elliot and Muller, 1983) has been suggested, but a thorough understanding of their differentiation, distribution, and roles during CNS development has been lacking in any simple invertebrate nervous system.

To what extent do glial substrates determine the major axon tracts in the developing CNS? How is the prepattern of glial pathways established? How do neurons interact with this glial prepattern in deciding which way to grow? The developing CNS of the Drosophila embryo has emerged as an excellent model system in which to study these questions because it is amenable to both a cellular and molecular genetic analysis. Many mutants have already been isolated and numerous genes cloned that are involved in neuronal development. Moreover, 2 well-characterized nuclear proteins are expressed in subsets of embryonic glia: single-minded and fushi tarazu (Doe et al., 1988; Thomas et al., 1988).

The work presented here demonstrates that at least 3 major classes of glia are present early in the development of the Drosophila CNS and appear to form an ordered pattern before the extension of the first neuronal growth cones. Based on extensive ultrastructural analysis of these glia and the first neuronal growth cones that interact with them, we suggest that the early glia in the developing Drosophila CNS may serve a guidance function for the major longitudinal and commissural axon tracts, and for at least one of the major peripheral nerve roots, the intersegmental nerve. This proposed role for insect glia in the formation of axon pathways is remarkably similar to that proposed for vertebrate glia, and this opens the way for a molecular genetic analysis of their function in this simple model system.

\section{Materials and Methods}

Fixation. After mechanical dechorionation, Drosophila embryos were prefixed for $10 \mathrm{~min}$ in heptane previously equilibrated with an equal volume of $25 \%$ glutaraldehyde and $2 \%$ acrolein (after Zalokar and Erk, 1977). Embryos were then blotted dry and immersed for $1 \mathrm{hr}$ in primary fixative containing $2 \%$ paraformaldehyde, $2.5 \%$ glutaraldehyde, and $0.5 \%$ 

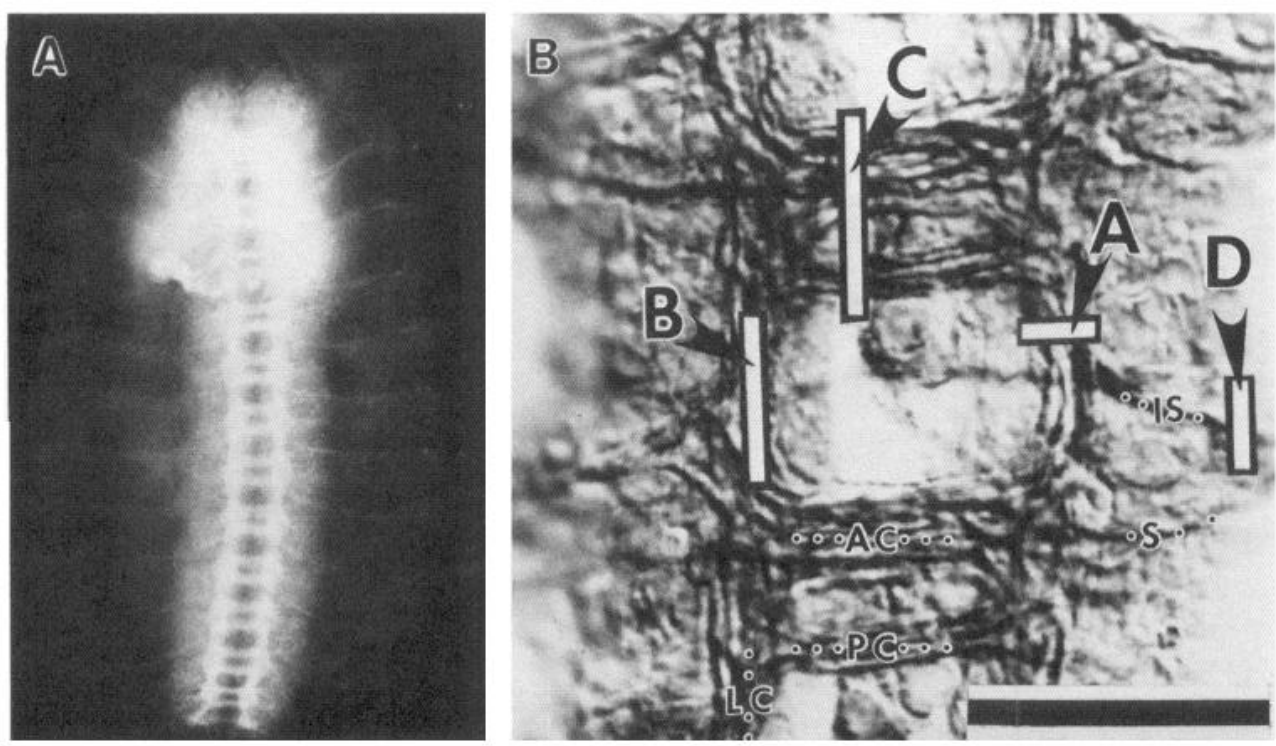

Figure 1. The embryonic axon scaffold of the Drosophila CNS at $12 \mathrm{hr}$ of development. The whole mount in $A$ is labeled with an antibody to HRP (Jan and Jan, 1982) and the dissection in $B$ with an antibody to tubulin, to demonstrate the organization of axon bundles during development. The axon scaffold in each segment consists of a bilateral pair of longitudinal connectives $(L C)$, an anterior and posterior commissure $(A C, P C)$, and 2 bilateral pairs of peripheral nerve roots, the segmental $(S)$ nerve and the intersegmen$\operatorname{tal}(I S)$ nerve. The letters beside the bars in $B$ represent the position of micrographs shown in Figure 2. Scale bars: $A, 150 \mu \mathrm{m} ; B, 20 \mu \mathrm{m}$.

dimethylsulfoxide in cacodylate buffer, $\mathrm{pH}$ 7.3. The vitelline was removed with electrolytically sharpened tungsten needles while in primary fix. Tissue was then osmicated, en bloc-stained in uranyl acetate, dehydrated, and embedded in Epon-Araldite.

Embryo staging. Fixed and embedded embryos were staged according to Campos-Ortega and Hartenstein (1985). However, because ultrastructural morphogenesis of the CNS occurs more rapidly than do gross features, stage subdivisions between 9:30 and $11 \mathrm{hr}$ of development (stages 12 and 13) are based upon CNS morphology.

Sectioning and electron microscopy. Sectioning and staining of serial ultrathin $(70-90 \mathrm{~nm})$ sections were performed as described by Stevens and Trogadis (1984). Material was examined and photographed on a Philips EM 410 electron microscope. Our analysis is based upon complete series through 6 segments of embryonic Drosophila nervous system from 4 embryos, 9:30-11 hr of development, as well as nonserial analysis of 33 embryos, from 8 to $14 \mathrm{hr}$ of development (sampling all stages between 11-15).

Computer-assisted reconstruction. Three-dimensional reconstructions were generated on a computer system described in detail by Stevens and Trogadis (1984). John Stevens generously provided us access to his computer system.

\section{Results}

\section{Glia in the Drosophila CNS comprise several different subclasses}

As in the CNS of other insects, the major axon tracts of the Drosophila CNS (within the thorax and abdomen) are organized into a ladder-like scaffold. Each of the 3 thoracic and the first 8 abdominal neuromeres have 2 commissures (one anterior and the other posterior) crossing the midline to connect the 2 longitudinal connectives (Fig. $1 A$ ). In addition, 2 peripheral nerve roots exit each side of each CNS neuromere: a bilateral pair of segmental nerves (at the level of the anterior commissure) and a bilateral pair of intersegmental nerves (ISNs; posterior to the posterior commissure). There is also a small median nerve root that exits the CNS from the dorsal midline. Examination of any of these axon tracts during embryogenesis or later life reveals that the limits and position of each tract are marked by conspicuous non-neuronal cells. These cells either enwrap the entire axon tract or elongate to form part of the outer wall of the tract.

We examined 4 locations within the segmentally repeated scaffold of axon tracts in the Drosophila embryo (see Fig. 1B) with the electron microscope in order to characterize the structure of these non-neuronal cells. The ultrastructure of these locations is shown in Figure 2, $A-D$. In each location, we found prominent glia in close apposition to axonal surfaces. For example, the dorsal surface of the longitudinal connective is overlaid by pairs of electron lucent cells (Fig. $2 A$ ). There are 3 pairs of cells (from anterior to posterior in each hemisegment) covering nearly the entire length of each segment, except for a small gap at the level of the anterior commissure. These longitudinal cells form a single-cell-thick roof over the developing neuropil (Fig. 2B). Similarly, the dorsal roof of the anterior and posterior commissures also have characteristic but different non-neuronal cells covering them (Fig. $2 C$ ). This roof is formed by 3 pairs of cells located along the dorsal midline of each segment.

The cells covering the axon tracts are distinguishable from neurons by a number of criteria. Before morphological differentiation, these cells have an elongate shape, with a high surface area and long or irregular nuclear profiles. Additionally, they are located along the dorsal surface of the developing CNS, where the axon tracts will shortly form (Figs. $2 B ; 4, A-C$ ). Later in development, they acquire an electron-lucent cytoplasm with an extensive rough endoplasmic reticulum and extend long sheets of cytoplasm from the soma that envelop nerve fascicles or enwrap the neuropil (Figs. $2 A, 4 D$ ). We classify these cells as glia because of their close association with axons and neuropil, their apparent structural role in ensheathing and supporting neuronal processes, and their morphological similarity to both vertebrate and invertebrate glia (Peters et al., 1976; Radojcic and Pentreath, 1979).

In a manner reminiscent of the vertebrate nervous system, the peripheral nerve roots in Drosophila are also completely ensheathed by glial cells. In Figure $2 D, 2$ glial cells share in enwrapping the ISN.

\section{Glia develop a prepattern before the first growth cones}

Previous studies on the guidance of neuronal growth cones in the grasshopper CNS have shown that a glial cell at the base of the intersegmental (IS) nerve, called the segment boundary cell (SBC), is in position before the first growth cones pioneer the ISN. The surface of the SBC is recognized by growth cones of the $U$ and aCC neurons, which turn laterally upon the SBC surface to pioneer the ISN (Bastiani and Goodman, 1986). 


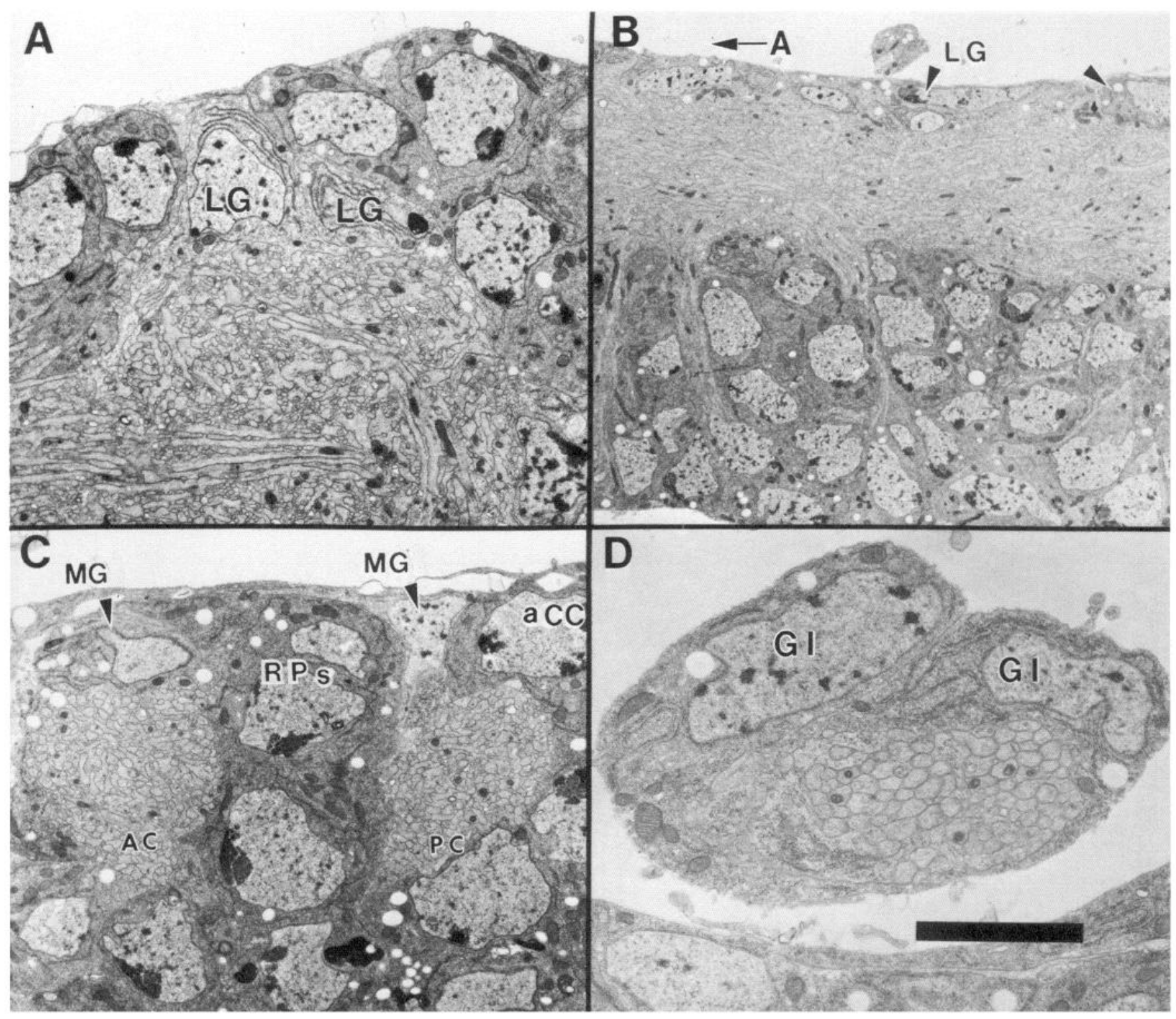

Figure 2. Glia morphology in the embryonic CNS at $11 \mathrm{hr}$ of development. $A$, In cross section through the longitudinal connective, 2 glial cells $(L G)$ form the dorsal roof of the developing neuropil. $B$, These glial cells are oriented along the length of the longitudinal connectives, as seen in this parasaggital section at the same stage of development (arrowheads). $C$, Glial cells $(M G)$ also cover the dorsal surface of the anterior (left) and posterior commissures, seen here in sagittal section. Posterior dorsal neurons like aCC also contribute to the dorsal surface of the posterior commissure, whereas the RP1 and RP3 neurons contribute to the cellular wall that separates the 2 commissures. $D$, The ISN ensheathed by 2 glial cells. Scale bars: $A$ and $C, 5 \mu \mathrm{m} ; B, 10 \mu \mathrm{m} ; D, 2.5 \mu \mathrm{m}$.

Moreover, when the SBC is ablated, the aCC neuron does not turn laterally along this pathway (Bastiani and Goodman, 1986).

In order to begin an analysis of whether glia play an analogous role in guiding the pioneers of the longitudinal and commissural tracts in the developing CNS, we examined the ultrastructure of the early developing CNS. We asked 3 questions. First, are the identity, position, and pattern of glial cells stereotyped during early development? Second, are the glia present in the positions of presumptive axon tracts before growth cones pioneer these tract? Third, do the first neuronal growth cones consistently contact the same glial cells as they pioneer specific axon tracts? Our studies demonstrate that many of the glia described above qualify on 2 or 3 of these criteria, which we consider more closely below.
Glia of the commissures. The anterior and posterior commissures are ensheathed by medially located pairs of cells on the dorsal surface of the nervous system (Fig. 3, $A, B$ ). The anterior pair, the midline glia anterior (MGA) cells, contributes to the dorsoanterior parts of the glial sheath of the anterior commissure. The middle pair, the midline glia middle (MGM) cells, separates the 2 commissures (as do the cell bodies of the RP1 and RP3 neurons, their most lateral neighbors), as well as ensheathing tracts in both commissures. The most posterior pair, the midline glia posterior (MGP) cells, contributes to the sheath of the posterior commissure, and also ensheathes axons headed to the median nerve.

The MG cells are good candidates for non-neuronal cells that guide the pioneers of the commissures. The MG cells are in 


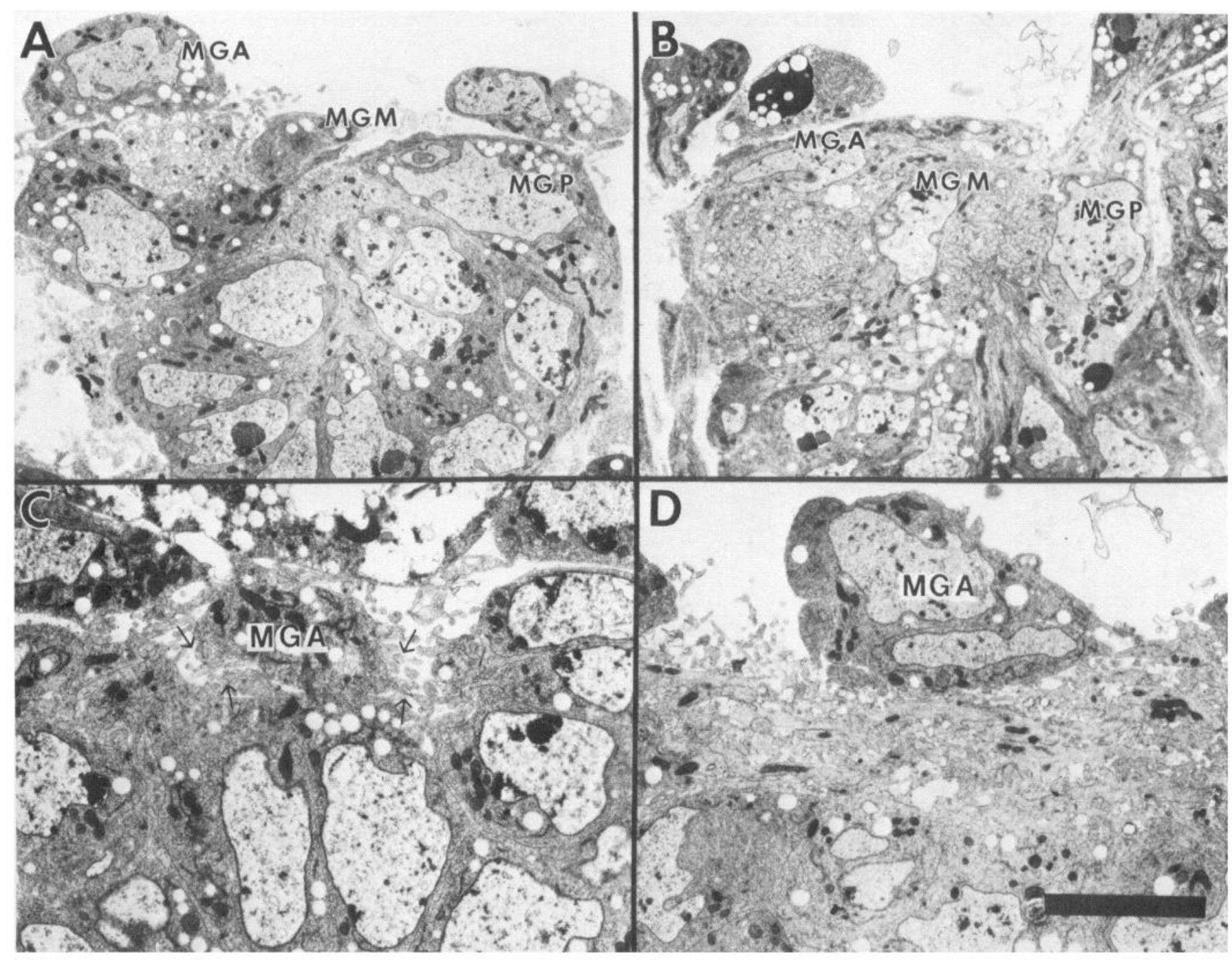

Figure 3. Development of glia over the commissures. $A$ and $B$, Sagittal sections through the commissures at 10 and $12 \mathrm{hr}$ of development. The anterior commissure at left is bordered dorsally by a pair of MGA cells and posteriorly by a pair of the MGM cells. The posterior commissure is bordered by the MGM and MGP cells. In cross section through the anterior segment at 9:40, before the anterior commissure is formed, growth cones and filopodia of neurons that will pioneer the commissure (arrows) are in contact with the MGA cells $(C)$. The same position at 10:30 hr is shown in $D$. Scale bars: $A$ and $B, 8 \mu \mathrm{m} ; C$ and $D, 6 \mu \mathrm{m}$.

their final position before the first axons pioneer the commissures. Figure $3 C$ shows the MGA cell sitting over the space where the anterior commissure will form, at 9:40 $\mathrm{hr}$ of development. The MGA cell is contacted by a number of growth cones and filopodia of neurons that will pioneer the anterior commissure. In the same location $45 \mathrm{~min}$ later in development, large numbers of axons course under the MGA cells (Fig. 3D). At this stage of development, the intracellular ultrastructure of glia is similar to the cytoplasm of neurons. Early glia are distinguished from neurons by their symmetric morphology (oval or cigar shaped, in contrast to the polarized structure of the neuron) and their position apposing presumptive nerve tracts in development.

Later in development, the MG cells acquire many of the morphological properties of ensheathing glia. As axonogenesis progresses, the MG cells increase their surface area and contact more commissural axons. In Figure $3 A$, during early axonogenesis, the MG cells are rounded and enclose the axon tracts of the commissures. Two hours later (Fig. $3 B$ ), large cytoplasmic sheets grow out of the MG cell bodies to ensheath commissural axons. As glia differentiate, their cytoplasm becomes more electron lucent than that of neurons.

Glia of the longitudinal connectives. The glia that lie on the dorsal surface of the longitudinal connective are well placed to guide the growth cones that pioneer the longitudinal axon tracts. Their development is examined in Figure 4. Three pairs of glia form the roof of the longitudinal tract before any axon extension occurs (8-9:45 hr). In concert with ongoing germ band shortening, glia are initially $10-14 \mu \mathrm{m}$ long, and shorten to $7-9 \mu \mathrm{m}$ long during development. The ventral side of the glial canopy is open extracellular space that is later occupied by the developing neuropil (Fig. 4A). The first axonal growth cones to appear, such as the vMP2 and pCC growth cones considered below, make extensive contact along the length of these glial cells.

After initial tract formation $(10 \mathrm{hr})$, ventrally located neurons project growth cones dorsally to the underbelly of the glial canopy. The glia experience extensive growth cone and filopodial contact, including filopodial insertion (Fig. 4, B, C). By this 


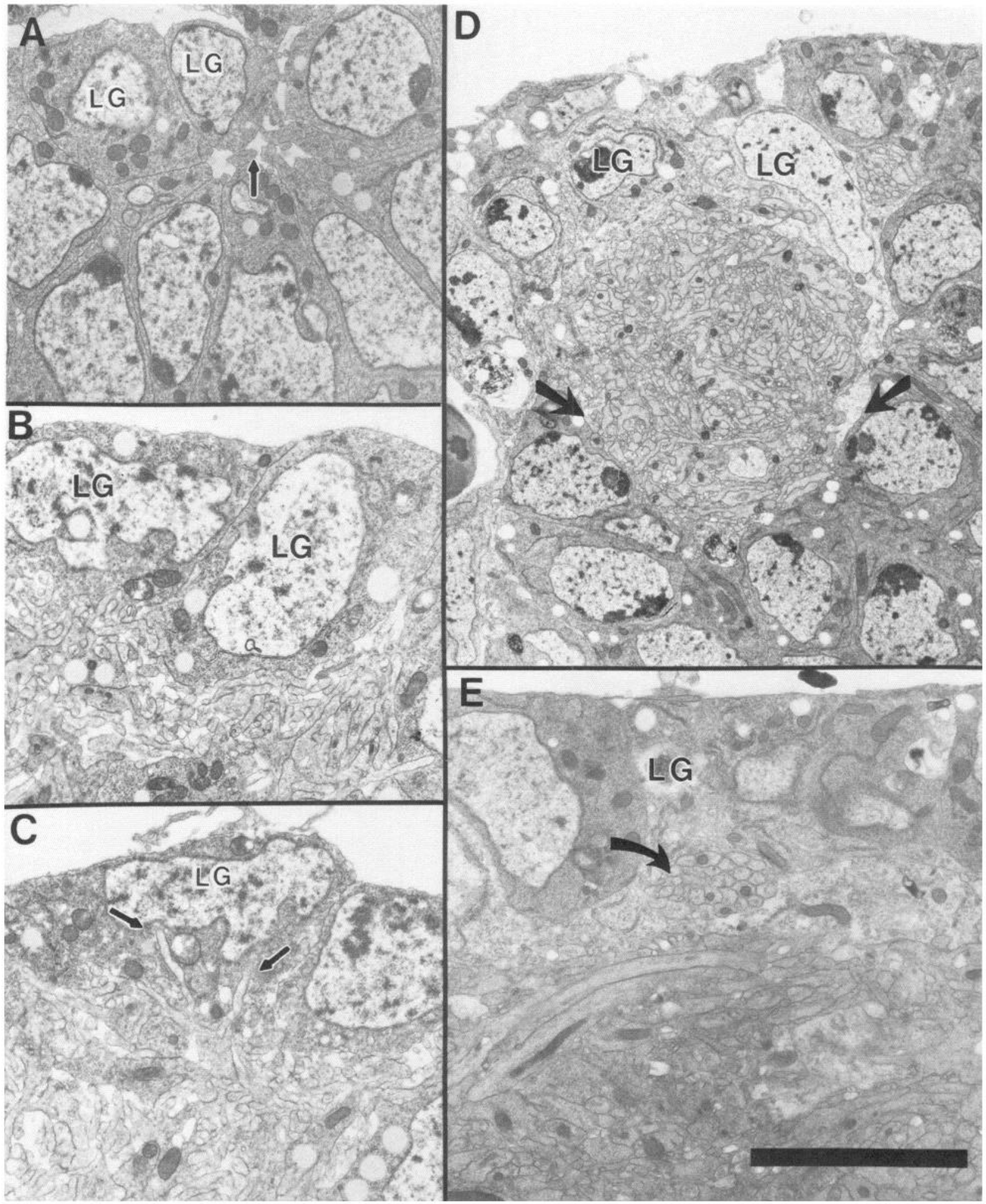

Figure 4. Development of glia over the longitudinal connectives. A, At $9.5 \mathrm{hr}$ of development, 2 glia form the dorsal boundary of the preformed pathway (arrow) through which the first axons will pass. $B$ and $C$, At $10 \mathrm{hr}$ of development, ventrally located neurons project numerous filopodia which interact with the overlying glia. Two large filopodial insertions are seen in $C$ (arrows). $D$, At $12 \mathrm{hr}$ of development, the dorsal glia begin to form large lamellae (arrows) to ensheath the entire neuropil. $E$, At $12 \mathrm{hr}$ in parasagittal section, a bundle of axons headed for the segmental nerve (arrow) is ensheathed by a dorsal glial cell. Scale bars: $4 \mu \mathrm{m}$ except $D, 7 \mu \mathrm{m}$. 
The Journal of Neuroscience, July 1989, 9(7) 2407

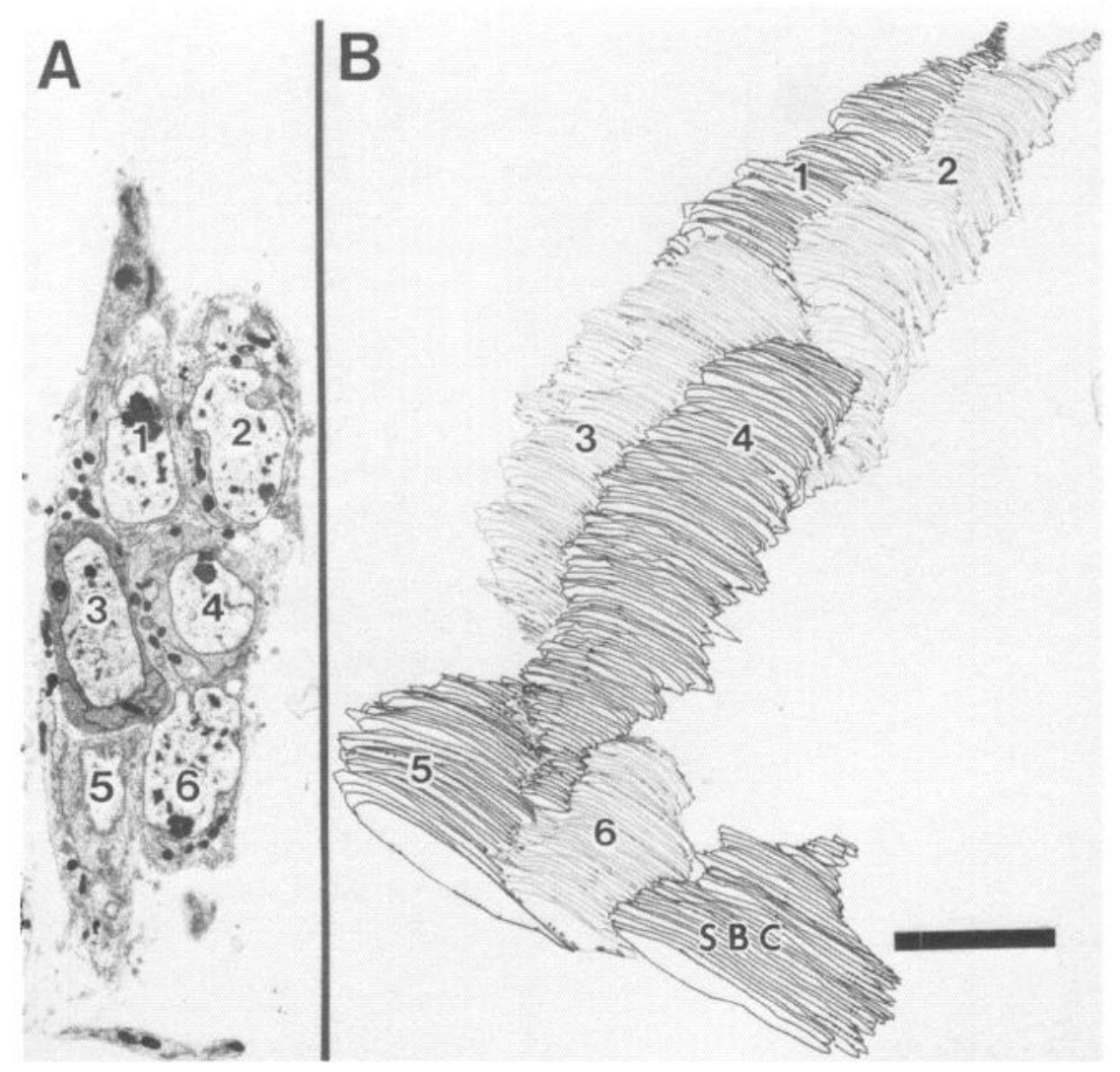

Figure 5. Organization of the glial scaffold over the CNS axon tracts. $A$, The profiles of 3 pairs of longitudinally oriented glia are seen in frontal section over the dorsal surface of the right longitudinal connective in a CNS at 10:15 hr. $B$, Reconstruction of the dorsal glia at the same stage generated from serial cross sections. Medial at left. The numbers 1-6 indicate identified longitudinal glial cells $(L G)$ of the longitudinal connective; the segment boundary cell $(S B C)$ is indicated. Scale bar, $4 \mu \mathrm{m}$.

time, the first growth cones have extended along the length of the dorsal glia, leading to the development of the initial axon fascicles adhering to the glial surfaces. By $12 \mathrm{hr}$ of development, dendritic and axonal processes begin to form a structured neuropil. At this stage, each pair of glia develops a large sheet of cytoplasm that encircles the neuropil from the lateral and medial sides (Fig. 4D). The glia will also envelop small fascicles of axons headed to the commissures or peripheral nerves (Fig. $4 E$ ).

From serial section reconstructions of 6 segments between 9:30 and $11 \mathrm{hr}$ of development, we have verified that the first dorsal glia are not only consistent in morphology and position, but are also individually recognized by neuronal growth cones. The 3 pairs of glia over the longitudinal connective provide a canopy from behind the anterior commissure to the posterior end of the segment. They can be visualized in a frontal section that grazes the surface of the CNS (Fig. $5 A$ ) and in a serial reconstruction of a segment at 10:15 hr (Fig. $5 B$ ).

Glia of the ISN. The origin of the ISN is marked by the SBC, which is the first of a string of glial cells oriented along the ISN as it projects to the periphery. Before axonogenesis the SBC is a compact, oblong cell oriented obliquely to the long axis of the CNS. This morphology is maintained in early axonogenesis (Fig. $6 \mathrm{~A}$ ) but differentiates at the same time the glia of the longitudinal tract do. The SBC becomes more elongate and enwraps the ISN. In a frontal plane of section (Fig. 6B), the SBC surrounds the ISN from the longitudinal tract just before exiting the CNS.

\section{Glia are specifically recognized by neuronal growth cones}

Ultrastructural analysis of the embryonic CNS reveals that filopodia, growth cones, and the initial axon fascicles are intimately associated with glial surfaces during early neuronal de- velopment. Additionally, we observed that all 3 sets of glial cells described above are consistent in their number, position, and pattern. The morphology and position of these 3 classes are shown schematically in Figure 7. Each class is in an appropriate position at the appropriate time in development to provide relevant positional information to the growth cones of neurons pioneering the major axon tracts of the CNS. If these glia provide positional information, then one would expect that identified neurons would respond in a consistent manner every time specific glia were encountered. For example, an axon-glia contact might be expected to be as specific as the axon-axon fasciculation choices seen in Drosophila (Bastiani et al., 1984; Thomas et al., 1984; Jacobs and Goodman, 1989).

Recognition of longitudinal glial cell LG5. From our reconstructions of pioneer neurons during the development of the longitudinal tract, we discovered that the growth cones of some identified neurons are consistently in apposition with the same glial cell during axon extension. These contacts are characterized by broad areas where apposing membranes are less than $10 \mathrm{~nm}$ from each other. Such contacts have not been described with high-resolution electron microscopy in Drosophila. The first neuron-glial contact during the formation of the longitudinal tract occurs as the growth cones of the pCC and vMP2 neurons extend anteriorly. These growth cones extend in tandem through the developing neuropil of their own segment to contact the longitudinal glial cell LG5 of the next segment. This is the first cell body that the growth cone of vMP2 adheres to, and vMP2 generally contacts LG5 more extensively than does the pCC growth cone. First contact is made with LG5 at $10 \mathrm{hr}$ (Fig. 8A). The growth cones flatten over all the available LG5 surface as they extend anteriorly (Fig. $8 B$ ) until they reach the MP1/dMP2 


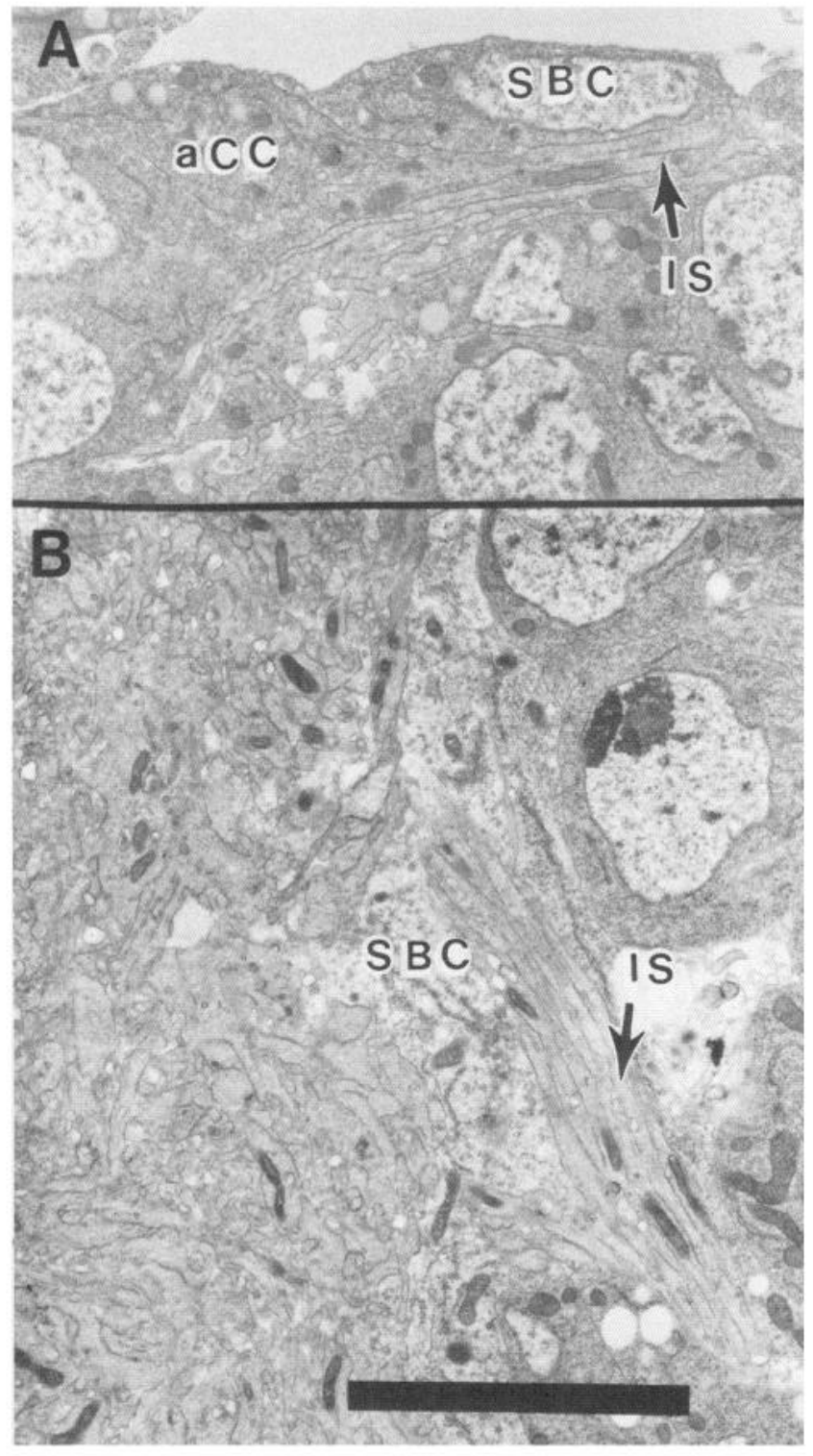

Figure 6. The intersegmental $(I S)$ nerve forms along the segment boundary cell $(S B C)$. The $\mathrm{SBC}$ is shown in cross section $(A)$ and frontal section $(B)$ with medial to the left. The SBC is relatively compact at $10: 15 \mathrm{hr}$ when only a few axons (aCC, the Us) form the IS nerve $(A)$ but becomes more elongated later as more axons join this nerve $(B)$ at 10:45 hr. Scale bar, $4 \mu \mathrm{m}$.

fascicle of the next anterior segment (see Jacobs and Goodman, 1989). The reconstruction in Figure 9 shows growth cone and glial cell morphology at the moment of first contact of vMP2 and $\mathrm{pCC}$ with identified glial cell LG5.

Recognition of the ISN glial cell SBC. The SBC is a prominent glial cell positioned just lateral to the longitudinal axon tract, at the point of origin of the ISN. In both the grasshopper and the fly, the growth cones of the neurons that pioneer the ISN turn laterally on the surface of the SBC, suggesting that recognition of and lateral extension along the SBC guides the growth cones in their proper projection towards the periphery (see Fig. 6). This has been verified experimentally in the grasshopper (Bastiani and Goodman, 1986). After ablation of the grasshopper SBC, axons that would normally turn into the ISN continue instead within the longitudinal connective.

\section{Discussion}

In this paper we describe 3 prominent classes of glial cells that appear to play an active role in the formation and development of the major axon tracts of the Drosophila CNS. Three pairs of midline glia ensheath the anterior and posterior commissures. Three other pairs of longitudinally oriented glia in each hemisegment ensheath the longitudinal connective, and peripheral nerve glia ensheath the nerve roots of the ISN. All 3 of these groups of glia are in the position of the axon tracts before the neuron pioneers have extended growth cones. In both the longitudinal tract and the ISN, it is the specific adhesion of pioneer growth cones to the surface of a remote glial cell that appears to guide the pioneer axon tract beyond the border of its own segment or into the PNS. Later in development, the same glia receive extensive filopodial contact from the growth cones of neurons immediately before they turn onto their appropriate axon tracts. When the dendritic neuropil begins to develop, the same glia extend cytoplasmic sheets to enclose the developing neuropil circuitry. The morphological differentiation of these glia reflects a change in their role in CNS morphogenesis. Early in morphogenesis, cell shape and position suggest that glia provide positional information and a substrate for axon extension. Subsequently, glia develop their elaborate cytoplasmic extensions, suggestive of a role in ensheathment and support of the neuropil.

Although these 3 groups of glia differentiate in a similar manner, they arise from different origins (J. R. Jacobs, Y. Hiromi, and C. S. Goodman, unpublished observations). For example, the glia of the longitudinal connective are the progeny of a lateral glioblast that arises out of the neuroblast layer of the $5 \mathrm{hr}$ embryo (Doe et al., 1988; Jacobs et al., 1989). This glioblast expresses the homeobox protein fushi tarazu (Doe et al., 1988) which can be used to monitor the differentiation of its progeny (Jacobs et al., 1989).

The glia of the commissures (the MG cells), on the other hand, arise from the mesectoderm of the midline neuroepithelium and express another nuclear protein, single minded ( $\mathrm{sim}$ ) (Crews et al., 1988). The sim protein is first expressed in a continuous medial stripe of cells at $4 \mathrm{hr}$ of development after gastrulation is complete and as the NBs begin to delaminate from the ectoderm. Six of the sim-positive cells in each segment become the MG cells, and they continue to express high levels of sim protein. The other cells become the median neuroblast (MNB), several other midline precursor cells, and other unidentified non-neuronal cells, all of which turn off their expression of the sim protein. Thus, by $10 \mathrm{hr}$ of development, the only 6 cells in each segment that express the sim protein are the $6 \mathrm{MG}$ cells (Crews et al., 1988; Jacobs et al., 1989).

In this paper, we have shown that the interactions of the pioneering growth cones with the early scaffold of glial cells is highly specific and stereotyped from segment to segment and embryo to embryo. These specific cell interactions appear to be mediated by specific contact events and are among the first signs of cell specificity during neuronal development. The participation of glia in the functional and spatial organization of early axon tract formation is consistent with the blueprint hypothesis as initially proposed for the development of the vertebrate spinal cord (Singer et al., 1979). These data are also consistent with and elaborate upon another hypothesis for pathway formation, the labeled pathways hypothesis (Goodman et al., 1983), as initially proposed for the development of the insect CNS. 


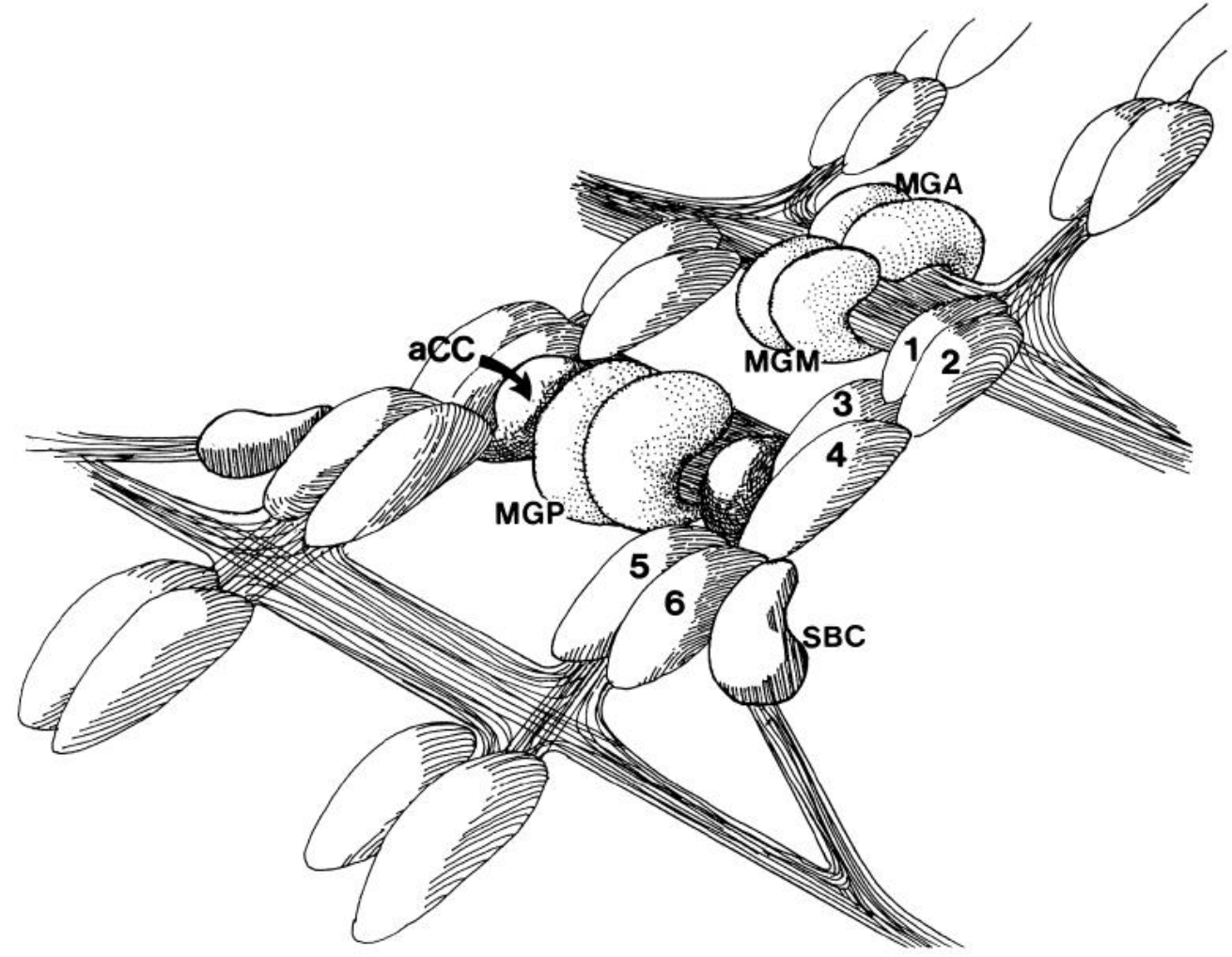

Figure 7. Schema of the axon tract glia present at initial axon outgrowth. Three classes of glia overlie 3 major axon tracts. The anterior and posterior commissures are demarcated by 3 pairs of midline glia (anterior, middle, and posterior: $M G A, M G M, M G P$ ). The longitudinal tracts have 3 pairs of longitudinal glia per segment (LG1-LG6) with a gap at the anterior commissure. Finally, the ISN is marked by the SBC the most proximal of the peripheral nerve glia.
The labeled pathways hypothesis was proposed to account for the remarkable specificity that growth cones display for specific axon pathways. The hypothesis predicts that growth cones choose specific axon bundles by their specific affinity for recognition molecules expressed on these axons. The question arose, however, as to how the very first axons in the CNS found their way before the first axon pathways had formed. The data pre- sented here suggest that just as the subsequent axon fascicles must be labeled by distinguishing markers, so the initial glial pathways must have some surface (and/or secreted) molecular heterogeneities that allow the first growth cones to distinguish among them. Clearly, the number of different glial markers might be quite small, but nevertheless, the clear choices made by the first growth cones suggest some specific guidance cues, and they

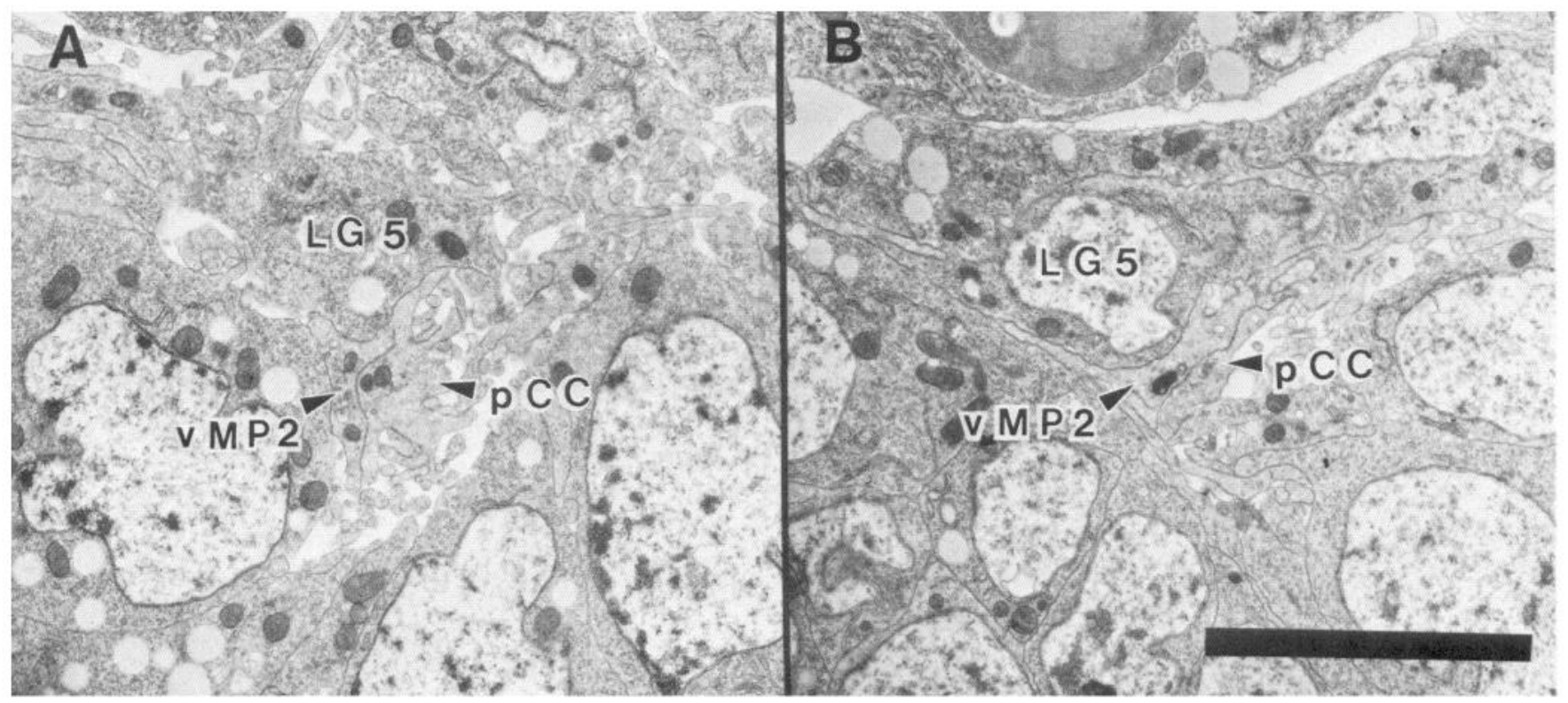

Figure 8. Identified glia: glial recognition by the growth cones of vMP2 and pCC. $A$, At $10 \mathrm{hr}$ of development, the growth cones of vMP2 and pCC encounter the most posterior dorsal glial cell of the anterior segment, LG5. B, At 10:15 hr, the growth cones of vMP2 and pCC have become flattened over the surface of the same glial cell. Scale bar, $4 \mu \mathrm{m}$. 


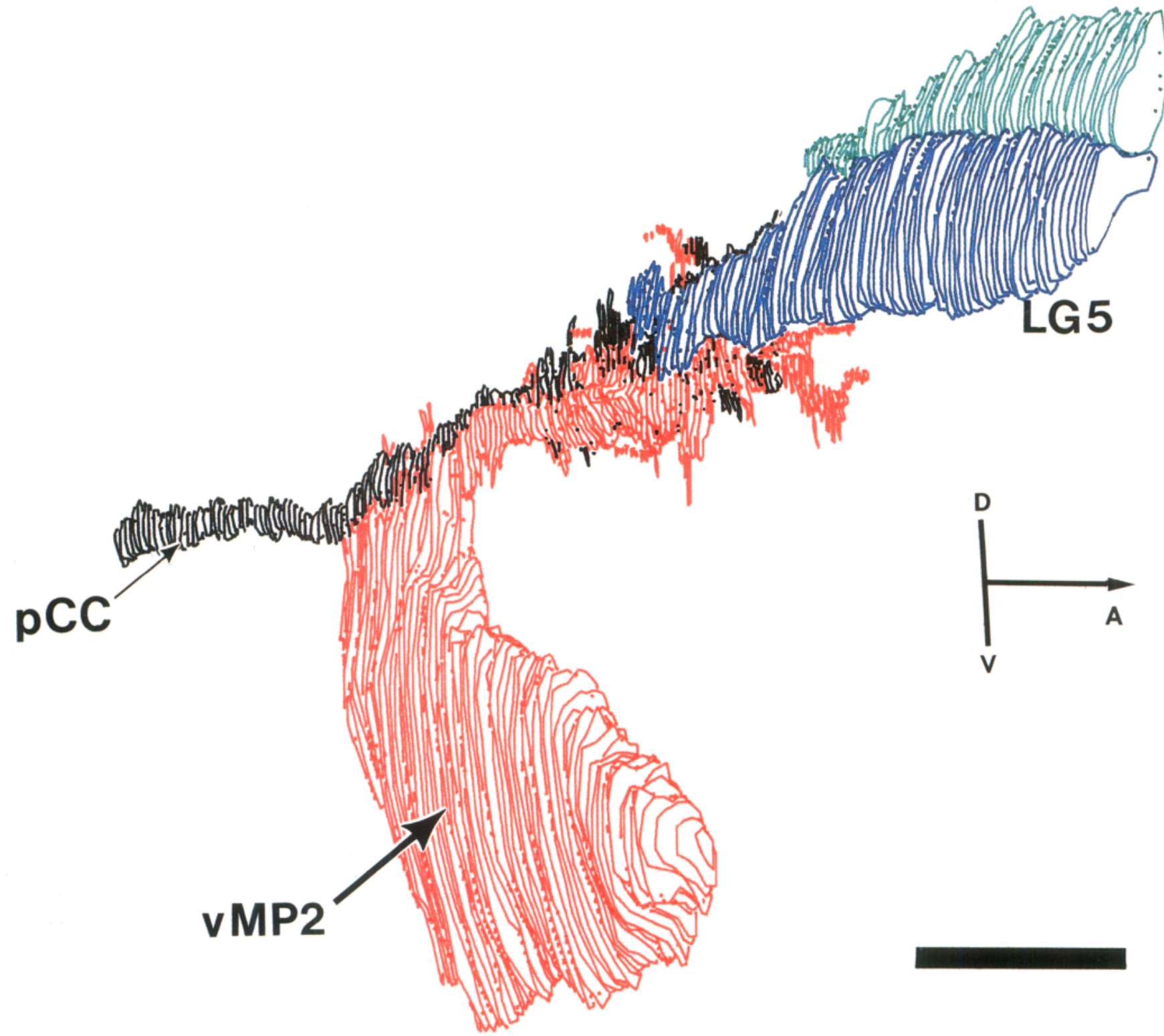

Figure 9. Recognition of glia LG5 by vMP2 and pCC. Reconstruction from serial electron micrograph cross sections at $10 \mathrm{hr}$ of development. Scale bar, $4 \mu \mathrm{m}$.

implicate the surface of the glia as one major source of these cues.

Many of the pioneering growth cones in the developing CNS make numerous filopodial insertions into the dorsal glia. Almost all filopodial insertions are directed into glia directly above the neuron's cell body shortly after axonogenesis, and they rarely occur after the growth cone has turned to extend in one of the longitudinal tracts. Filopodial insertions have been reported in other instances of cell-cell recognition, such as neuron-neuron recognition in the grasshopper (Bastiani and Goodman, 1984). It is possible that neurons at the initial stages of growth cone extension are reading positional information from the overlying glia before making their first pathway decisions and that filopodial insertions might be involved in these interactions. After the major axon tracts have formed $(10: 30 \mathrm{hr})$, filopodial insertions into glia are rarely seen.
The recognition events between individual growth cones and specific glial cells as described in this paper are limited to the initial period of axonogenesis and pathway formation. Once the scaffold of axon pathways has formed, later growth cones fasciculate with existing axon pathways and no longer interact in this way with the glia. After $11 \mathrm{hr}$, the neuropil begins to develop. At this time, the glia begin morphological differentiation into their mature types. Peripheral nerve glia, such as the SBC, ensheath the axons in the IS nerve in a manner reminiscent of Schwann cells. Sheath glia in insects have been previously defined as a distinct class of glia by Sohal et al. (type III; 1973) and Strausfeld (type 4; 1976). The glia of the longitudinal tracts develop in a similar manner, forming large lamellar sheets to enclose the entire neuropil. These cells likely perform a nutritive and isolating function like oligodendroglia. Strausfeld includes these cells as a variety of sheath glia because of their similar 
function and morphology. Their similar development and shared role in tract formation support the concept of their functional similarity.

The early glia in the Drosophila CNS provide a nearly complete scaffold or template for the formation of the major axon tracts before any growth cones have appeared. Templates of this variety have been described in the development of a number of nervous systems, with glia intimately involved in many of them. Glia provide a preformed path for the formation of the corpus callosum and anterior commissure of the mouse brain (Silver ct al., 1982). In the mousc, as well as the fly, the glia are oriented along the axis of the preformed path, and the growth cones extend along the long axis of the glia. During the development of the spinal cord of the newt (Singer et al., 1979), the trout tail (Gerardine and Singer, 1982), and the optic nerve (Silver and Rutishauser, 1984), the neuroepithelium and undifferentiated glial cells create a pattern of open channels through which pioneering axons extend. Although the open channel is clearly oriented to create a preformed path, the cells lining the channel are not oriented as they are in the mouse callosum or Drosophila scaffold. Nevertheless, a general feature of all preformed pathways is a preference for the growth cone to adhere to the cell membranes that form the perimeter of the pathway. If this adhesion is disrupted, tract morphogenesis is disturbed or prevented (Silver et al., 1982; Silver and Rutishauser, 1984).

Just as in Drosophila, so in grasshopper, glia are prominent components during the development of axon pathways in the CNS (Bastiani and Goodman, 1986). The cell bodies of grasshopper glia are placed laterally along the edge of the developing longitudinal axon tracts but extend numerous processes along the dorsal basement membrane over the location where the first longitudinal pathways form. Whereas in the fly the first growth cones confront an environment with small channels under a complete covering of glial cells, in the grasshopper the channels are larger and the glial covering incomplete, with exposed stretches of dorsal basement membrane (Bastiani et al., 1984). Nevertheless, the pioneering growth cones in the grasshopper and fly make similar pathway choices and establish homologous axon pathways (see companion paper, Jacobs and Goodman, 1989). Thus, where the first growth cones in Drosophila appear to interact primarily with the surfaces of the early glial cells in a rather confined environment, the homologous growth cones in the grasshopper interact with both the glia and the basement membrane in a more spacious environment. It seems likely, however, that common molecular mechanisms may be involved in guiding these first growth cones in both insects. Thus, the common element in both insects, the 3 different classes of glia described in this paper, are prime candidates for the source of these guidance cues.

\section{References}

Bastiani, M. J., and C. S. Goodman (1984) Neuronal growth cones: Specific interactions mediated by filopodial insertion and induction of coated vesicles. Proc. Natl. Acad. Sci. USA 81: 1849-1853.

Bastiani, M. J., and C. S. Goodman (1986) Guidance of neuronal growth cones in the grasshopper embryo. III. Recognition of specific glial pathways. J. Neurosci. 6: 3542-3551.

Bastiani, M. J., K. G. Pearson, and C. S. Goodman (1984) From embryonic fascicle to adult tracts: Organisation of neuropile from a developmental perspective. I. Exp. Biol. 112: 45-64.

Campos-Ortega, J. A., and V. Hartenstein (1985) The Embryonic Development of Drosophila melanogaster. Springer-Verlag, Berlin.

Crews, S. T., J. B. Thomas, and C. S. Goodman (1988) The Drosophila single-minded gene encodes a nuclear protein with sequence similarity to the per gene product. Cell 52: 142-151.

Doe, C. Q., Y. Hiromi, W. J. Gehring, and C. S. Goodman (1988) Expression and function of the segmentation gene fushi tarazu during Drosophila neurogenesis. Science 239: 170-175.

Elliot, E. J., and K. J. Muller (1983) Sprouting and regeneration of sensory axons after destruction of ensheathing glial cells in the leech central nervous system. J. Neurosci. 3: 1994-2006.

Gerardine, J., and M. Singer (1982) Axonal guidance in the neuroepithelium of the tail of the trout Salmo gairdneri. J. Exp. Zool. 219: 355-360.

Goodman, C. S., J. A. Raper, S. Chang, and R. Ho (1983) Grasshopper growth cones: Divergent choices and labelled pathways. Prog. Brain Res. 58: 283-296.

Hoyle, G. (1986) Glial cells of an insect ganglion. J. Comp Neurol. 246: 85-103.

Jacobs, J. R., and C. S. Goodman (1989) Embryonic development of axon pathways in the Drosophila CNS. II. Behavior of pioneer growth cones. J. Neurosci. 9: 2412-2422.

Jacobs, J. R., Y. Hiromi, N. H. Patel, and C. S. Goodman (1989) Lineage, migration and morphogenesis of longitudinal glia in the Drosophila CNS as revealed by a molecular lineage marker. Neuron (in press).

Jan, L. Y., and J. N. Jan (1982) Antibodies to horseradish peroxidase as specific neuronal markers in Drosophila and in grasshopper embryos. Proc. Natl. Acad. Sci. USA 79: 2700-2704.

Peters, A., S. L. Palay, and H. deF. Webster (1976) The Fine Structure of the Nervous System, Saunders, Philadelphia.

Radojcic, T., and V. W. Pentreath (1979) Invertebrate glia. Prog. Neurobiol. 5: 115-179.

Rakic, P. (1971) Neuron-glia relationship during granule cell migration in developing cerebellar cortex. A golgi and electron microscopic study in Macacus rhesus. J. Comp. Neurol. 141: 283-312.

Roitbak, A. I. (1983) Neuroglia. Fischer Verlag, Berlin.

Silver, J., and U. Rutishauser (1984) Guidance of optic axons in vivo by a pre-formed adhesive pathway on neuro-epithelial endfeet. Dev. Biol. 106: 485-499.

Silver, J., S. E. Lorenz, D. Wahlstein, and J. Coughlin (1982) Axonal guidance during development of the great cerebral commissures: Descriptive and experimental studies in vivo on the role of preformed glial pathways. J. Comp Neurol. 210: 10-29.

Singer, M., R. H. Nordlander, and M. Egar (1979) Axonal guidance during embryogenesis and regeneration in the spinal cord of the newt. The blueprint hypothesis of neuronal pathway patterning. J. Comp. Neurol. 185: 1-22.

Sohal, R. S., S. P. Sharma, and E. F. Couch (1973) Fine structure of the neural sheath, glia and neurons in the brain of the housefly, Musca domestica. Z. Zellforsch. Mikrosk. Anat. 135: 449-459.

Stevens, J. K., and J. T. Trogadis (1984) Computer-assisted reconstruction from serial electron micrographs: A tool for the systematic study of neuronal form and function. Adv. Cell. Neurobiol. 5: 341369.

Strausfeld, N. J. (1976) Atlas of an Insect Brain, Springer-Verlag, Berlin.

Thomas, J. B., M. J. Bastiani, C. M. Bate, and C. S. Goodman (1984) From grasshopper to Drosophila: $\Lambda$ common plan for neuronal development. Nature 310: 203-207.

Thomas, J. B., S. T. Crews, and C. S. Goodman (1988) Molecular genetics of the single-minded locus: A gene involved in the development of the Drosophila nervous system. Cell 52: 133-141.

Wigglesworth, V. B. (1959) The histology of the nervous system of an insect Rhodnius prolixus. II. The central ganglia. Q. J. Microsc. Sci. 100: 299-314.

Zalokar, M., and I. Erk. (1977) Phase partition fixation of Drosophila eggs. Stain. Technol. 52: 89-95. 